\title{
Coexpression of periostin and EGFR in patients with esophageal squamous cell carcinoma and their prognostic significance
}

This article was published in the following Dove Press journal:

OncoTargets and Therapy

18 August 2016

Number of times this article has been viewed

\author{
Wei Jia' \\ Wei Wang' \\ Chu-shu Ji' \\ Jun-yang $\mathrm{Niu}^{2}$ \\ Ya-jing Lv' \\ Hang-cheng Zhou ${ }^{2}$ \\ Bing $\mathrm{Hu}^{\prime}$ \\ 'Department of Medical Oncology, \\ ${ }^{2}$ Department of Pathology, Anhui \\ Provincial Hospital, Anhui Medical \\ University, Hefei, People's Republic \\ of China
}

Background: Both periostin (PN) and epidermal growth factor receptor (EGFR) can predict the prognosis of several carcinomas alone. However, coexpression of PN and EGFR in esophageal squamous cell carcinoma (ESCC) still remains unknown. We aimed to clarify their relationship with clinicopathological factors and prognostic significance of their coexpression in ESCC.

Patients and methods: In this single-center retrospective study, immunohistochemistry was performed to evaluate the expression of PN and EGFR in ESCC and paracarcinomatous tissues of 83 patients. The quantitative expression levels of PN and EGFR were examined in two ESCC and tumor-adjacent tissues. The levels of PN and EGFR expression were correlated with clinicopathological parameters by the $\chi^{2}$ or Kruskal-Wallis method. Spearman's rank correlation test was performed to determine the relationship between PN and EGFR expression levels. Kaplan-Meier and Cox regression analyses were used to detect the prognostic factors of disease-free survival (DFS) and overall survival (OS).

Results: The high expression of PN protein in ESCC tissues was significantly associated with tumor length $(P=0.044)$, differentiation grade $(P=0.003)$, venous invasion $(P=0.010)$, invasion depth $(P=0.007)$, lymphatic metastasis $(P=0.000)$, and tumor stage $(P=0.000)$. The high expression of EGFR protein in ESCC tissues was only significantly related to lymphatic metastasis $(P=0.000)$, invasion depth $(P=0.022)$, and tumor stage $(P=0.000)$. Kaplan-Meier analysis showed that high expression of PN was closely correlated to reduced OS $(P=0.000)$ and DFS $(P=0.000)$, which was consistent with EGFR expression. Cox regression analysis identified PN and EGFR as independent poor prognostic factors of OS and DFS in the ESCC patients $(P<0.05)$. Moreover, the risk of death for the ESCC patients with low expression of two biomarkers and high expression of single biomarker was 0.243 times $(P=0.000)$ and 0.503 times $(P=0.030)$, respectively, than that for patients with high expression of two biomarkers.

Conclusion: PN and EGFR are related to miscellaneous clinicopathologic characteristics. Coexpression of PN and EGFR is more closely to be of predictive value on ESCC development and progression, which may offer a novel and potential target strategy for ESCC treatment in the future.

Keywords: esophageal squamous cell carcinoma, periostin, epidermal growth factor receptor, prognosis

\section{Introduction}

Esophageal carcinoma, one of the virulent upper gastrointestinal tract malignant tumors, is the eighth most common incident cancer and sixth in lethal globally. ${ }^{1}$ Esophageal adenocarcinoma is the most predominant type of esophageal cancer in America and Europe, while more than $80 \%$ of patients with esophageal cancer in 
the developing countries have esophageal squamous cell carcinoma (ESCC)., ${ }^{2,3}$ Owing to the lack of effective tumor biomarkers or characteristic symptoms for early diagnosis, a large number of the ESCC patients are diagnosed at late advanced stages. Recently, albeit noticeable improvements in surgical technique, chemotherapy, and radiotherapy, the prognosis for patients with ESCC remains unsatisfactory, in which the overall 5-year survival rate after radical resection of esophageal carcinoma ranges from $15 \%$ to $25 \%$. $^{1,2,4}$ Thus, it is urgent to find more effective molecular biomarkers of progression and recurrence in ESCC for targeted therapy.

Periostin (PN), as a soluble and secreted extracellular matrix protein, is highly expressed during embryonic development and injury or inflammation within adult organisms. ${ }^{5}$ Currently, the available literature has frequently detected that $\mathrm{PN}$ is also upregulated in various human malignant cancers, such as head and neck, thyroid, breast, lung, ovarian, colon, gastric, pancreatic, and liver. ${ }^{5-9}$ Some literature showed that PN played a significant role in biologic processes, including cell adhesion, proliferation, angiogenesis, tumor invasion, and metastatic growth. ${ }^{10}$ Additionally, PN embraces domains that can bind some integrins $(\alpha v \beta 3$ and $\alpha v \beta 5)$ and their combination can activate downstream proteins by interacting with some cell surface receptors. ${ }^{5,11}$ Current research has indicated that PN expression is upregulated in cells that highly expressed both epidermal growth factor receptor (EGFR) and mutant p53 compared to control cells that highly expressed EGFR or mutant p53 alone. Meanwhile, PN protein expression in in vitro cells was decreased by inhibiting EGFR or restoring wild-type p53 signaling, suggesting that PN expression was modulated mechanistically by activating EGFR signaling and p53 mutation. ${ }^{11}$ Besides studies of PN in in vitro cells, PN might be used to detect preneoplastic lesions in ESCC xenograft tumors of mice. ${ }^{12}$ Up to now, few have reported the prognostic significance of $\mathrm{PN}$ and EGFR in patients with ESCC alone. According to the reaction of $\mathrm{PN}$ and EGFR in in vitro cells mentioned earlier, whether the coexpression of PN and EGFR as prognostic factors was superior to either PN or EGFR as a single marker for the ESCC patients still remains unknown.

The EGFR, also named c-erb-B1, is a $170 \mathrm{kDa}$ transmembrane glycoprotein and belongs to receptor tyrosine kinases family. ${ }^{13}$ EGFR participates in many aspects of cell biology and can be activated not only by ligand-induced signaling but also by many more protein kinases, such as integrins, cytokine receptors, and adhesion receptors. ${ }^{14}$ Existing evidence suggests that increased expression of EGFR is involved in $50 \%-70 \%$ of esophageal cancer cases. ${ }^{13,15}$
However, the results of EGFR tyrosine kinase inhibitors such as nimotuzumab used in the treatment of esophageal cancer remain poor. Liang et $\mathrm{al}^{16}$ demonstrated that the survival rate of 3 years among 42 patients with ESCC was only $26 \%$ after using nimotuzumab and radiotherapy.

Since EGFR and PN are both involved in multiple aspects of carcinogenesis, it is assumed that coexpression of EGFR and PN may affect the prognosis of the ESCC patients, which potentially offers a novel therapeutic target strategy. To testify the abovementioned hypothesis, the expression levels of EGFR and PN in 83 ESCC samples or corresponding paracarcinomatous normal tissues were observed by immunohistochemistry (IHC) in the present study. Moreover, the quantitative expression levels of $\mathrm{PN}$ and EGFR proteins in two fresh-frozen ESCCs and corresponding paracarcinomatous normal tissues were analyzed by Western blot analysis. Additionally, the survival analysis was performed by the Kaplan-Meier and Cox regression methods. All the above referred aspects aimed to demonstrate the expression of EGFR and PN in ESCC and its correlation with clinicopathological features and prognosis.

\section{Patients and methods Patients and tissue samples}

Eighty-three ESCC patients who underwent curative esophageal resection between April 2009 and June 2011 and two patients who underwent curative resection of ESCC in May 2016 at Anhui Provincial Hospital, affiliated to Anhui Medical University, were enrolled in our single-center retrospective study. No patients received treatment for the tumor prior to the operation, and histological diagnoses after operation were confirmed by pathology according to the seventh edition of the American Joint Committee on Cancer criteria. All patients who had prior malignancies or a second primary tumor were excluded. Clinical and pathological data were obtained retrospectively from medical records. Telephone follow-ups were performed every 3 months from the dates of surgery to death or to follow-up deadlines (September 2015). All clinical and pathological data were used to analyze the correlation with PN and EGFR expression levels. The written consent was obtained from all participants, and this study was approved by the Research Ethics Committee of Anhui Provincial Hospital, Anhui Medical University.

\section{Immunohistochemistry}

The expression levels of EGFR and PN protein in tumors and corresponding paracarcinomatous normal tissues were detected by IHC staining using a two-step method. Briefly, 
all formalin-fixed and paraffin-embedded esophageal cancer specimens were cut into sections of $4 \mu \mathrm{m}$ thickness by paraffin microtome. After deparaffinization and rinse, antigen retrieval was accomplished by heating specimen slices incubated in $\mathrm{pH} 6.0$ citrate buffer using a microwave oven. Endogenous peroxidases were blocked by using 3\% hydrogen peroxide for 5 minutes followed by washing three times in buffer. The sections were then incubated with primary antibodies against respective target proteins (PN antibody [ab92460; Abcam Inc, Shanghai, People's Republic of China], EGFR antibody [ZM-0093; Zhongshan Jinqiao Co., Beijing, People's Republic of China]) at a dilution of 1:150 at room temperature for 2 hours. After incubating with secondary antibody (mouse antirabbit IgG; Zhongshan Jinqiao Co.) at room temperature for 30 minutes, diaminobenzidine (Zhongshan Jinqiao Co., Beijing, People's Republic of China) and hematoxylin were performed to visualize the staining of target proteins. The positive and negative controls were well-known positive tissue sections and slides processed with phosphate-buffered saline, respectively.

The stained slices were assessed independently by two experienced pathologists who were blinded to the diagnosis and prognosis. The IHC expression levels of PN and EGFR were scored partially using the percentage of positive cell and the staining intensity published in previous article. ${ }^{17}$ Grading of tumor cells' staining intensity was as follows: negative, 0 ; light yellow, 1; brownish-yellow, 2; and brown, 3. Grading of stain-positive tumor cells' distribution was as follows: stain-positive cells $<5 \%, 0$; stain-positive cells $5 \%-25 \%, 1$; stain-positive cells $26 \%-50 \%, 2$; and stainpositive cells $>50 \%, 3$.

$$
\text { Score }=\text { Grade in percentage of positive cell }
$$$$
\times \text { Grade in strain intensity }
$$

We regarded score $\geq 4$ as the high expression group and the low expression group contained negative and low expressive samples.

\section{Western blot analysis}

Fresh-frozen tumor tissues and corresponding tumor-adjacent normal tissues of two ESCC patients were lysed using cell lysate buffer. After separation by $10 \%$ sodium dodecyl sulfate polyacrylamide gel electrophoresis, the protein extraction was transferred to polyvinylidene fluoride membranes (EMD Millipore, Billerica, MA, USA). The membranes were blocked by using $5 \%$ nonfat milk solution for 2 hours at room temperature and then incubated with primary antibodies
(PN antibody [1:800; Abcam Inc], EGFR antibody [1:250; Zhongshan Jinqiao Co.], and beta-actin antibody [1:400; Zhongshan Jinqiao Co.]) at $4{ }^{\circ} \mathrm{C}$ overnight. The membrane was then incubated with antirabbit or antimouse secondary antibodies conjugated with horseradish peroxidase $(1: 20,000$; Zhongshan Jinqiao Co.) for 1 hour at room temperature followed by washing with Tris-buffered saline Tween-20. Finally, the membrane was developed using the enhanced chemiluminescence systems. The computerized densitometer (ImageJ Launcher; Broken Symmetry Software, NIH, Bethesda, MD, USA) was used to quantify band intensities, and beta-actin was used to confirm that an equal amount of protein was loaded in each track.

\section{Statistical analysis}

Data were analyzed using SPSS Version 13.0 (SPSS Inc., Chicago, IL, USA) software. $P$-value $<0.05$ was considered as a significant difference. According to PN or EGFR status, the patients were categorized into high- or low-expression groups. $\chi^{2}$ or Kruskal-Wallis test was used to detect a relationship between clinicopathologic parameters and $\mathrm{PN}$ or EGFR expression. The correlation between survival records and expression levels of proteins was conducted using the Kaplan-Meier curve and log-rank test. Multivariate analysis was assessed by using the Cox regression model.

\section{Results}

\section{PN and EGFR expression in ESCC and adjacent normal esophageal tissues}

In our study, a total of 83 primary ESCC sections and paired paracarcinomatous normal esophageal tissues were evaluated by IHC. The IHC scores in ESCC tumor were significantly higher than those in tumor-adjacent normal tissues $(P=0.000$; Figure 1). The staining scores in all the normal esophageal sections were $<3$. In this study, a high expression of PN in the tumor tissues was detected in 59 of 83 (71.1\%) patients, whereas a low expression was observed in 24 of $83(28.9 \%)$ patients. The high expression of PN was mainly detected in tumor cytoplasm and extracellular matrix around ESCC cells (Figure 2A-C), while normal esophageal tissues showed a weak or no staining.

The EGFR protein was also examined in 83 primary ESCC sections and paired paracarcinomatous normal esophageal tissues by IHC. The high expression of EGFR was detected in 54 of 83 (65.1\%) patients, whereas low expression was observed in 29 of 83 (34.9\%) patients. The EGFR expression in tumor-adjacent normal tissues was significantly lower than that in ESCC tissues $(P=0.000$; Figure 1). The expression of EGFR was mainly detected in 


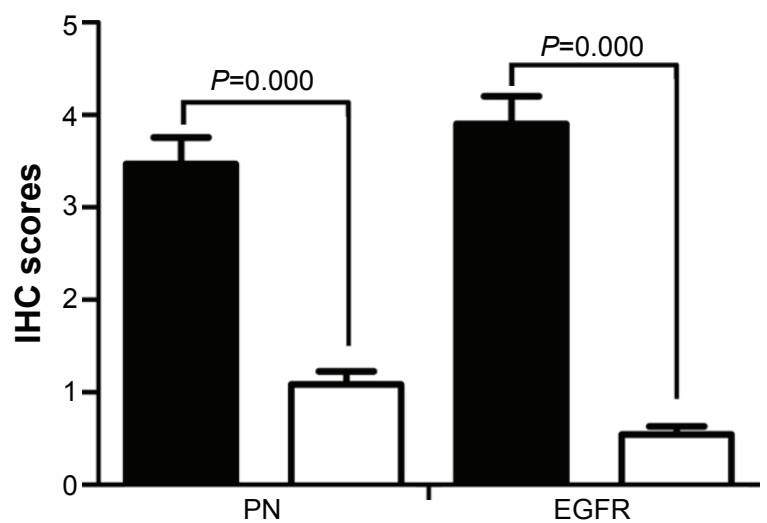

Figure I IHC scores of PN and EGFR expression in ESCC and tumor-adjacent normal tissues.

Notes: Proteins expressed in ESCC tumors (black bar graph), and proteins expressed in tumor-adjacent normal tissues (white bar graph). Error bars are SEs. Abbreviations: IHC, immunohistochemistry; PN, periostin; EGFR, epidermal growth factor receptor; ESCC, esophageal squamous cell carcinoma.

ESCC cell membrane (Figure 2D-F), and the staining scores in normal esophageal sections were almost $<2$.

Additionally, in order to further confirm the trends of expression, the expression levels of $\mathrm{PN}$ and EGFR proteins in two fresh-frozen ESCC and corresponding paracarcinomatous normal tissues were analyzed by Western blot analysis. The expression levels of PN and EGFR in ESCC were higher than those in the corresponding tumor-adjacent normal tissues, which was similar to the results of IHC analysis (Figure 3).

\section{Relationship between PN, EGFR expression, and pathological characteristics}

Based on immunohistochemical analysis, we compared the relationship between PN, EGFR expression, and pathological characteristics. As summarized in Table 1, the high expression of PN in ESCC tissues was significantly associated with tumor length $(P=0.044)$, differentiation grade $(P=0.003)$, venous invasion $(P=0.010)$, invasion depth $(P=0.007)$, lymphatic metastasis $(P=0.000)$, and clinical stage $(P=0.000)$. No significant association was observed between the high expression of PN and other clinicopathological parameters such as tumor location, gender, and age. In the present study, the high expression of EGFR protein in ESCC tissues was only significantly related to the lymphatic metastasis $(P=0.000)$, invasion depth $(P=0.022)$, and tumor stage $(P=0.000)$. There was no significant correlation between EGFR and other clinicopathological parameters.

\section{Correlation analysis of PN and EGFR}

The relationship between the high expression of EGFR and $\mathrm{PN}$ is shown in Table 2. The high expression levels of both PN and EGFR were detected in 41 of 83 (49.4\%) patients, while low or no expression was observed in 23 of $83(27.7 \%)$ patients. The results also showed that a positive correlation occurred in the high expression levels of EGFR and PN ( $r=0.531 ; P=0.001$; Table 2).
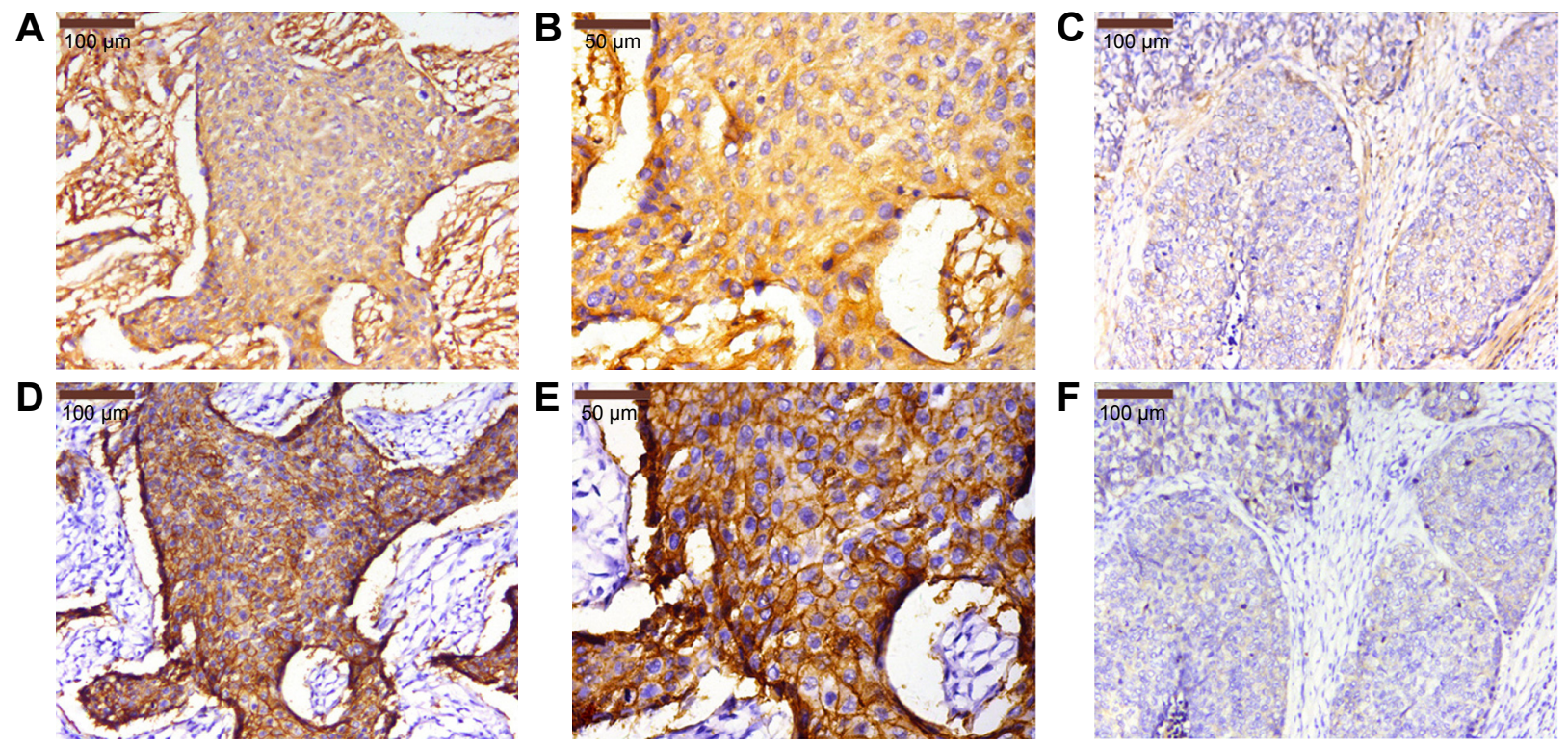

Figure 2 Expression levels of PN and EGFR in ESCC tissues by IHC.

Notes: (A and B) High expression of PN in ESCC tissues. (C) Low expression of PN in ESCC tissues. (D and E) High expression of EGFR in ESCC tissues. (F) Low expression of EGFR in ESCC tissues. ( $\mathbf{A}$ and $\mathbf{D})$ is $\times 200$ magnification, ( $\mathbf{B}$ and $\mathbf{E})$ is $\times 400$ magnification, and $(\mathbf{C}$ and $\mathbf{F})$ is $\times 200$ magnification.

Abbreviations: PN, periostin; EGFR, epidermal growth factor receptor; ESCC, esophageal squamous cell carcinoma; IHC, immunohistochemistry. 


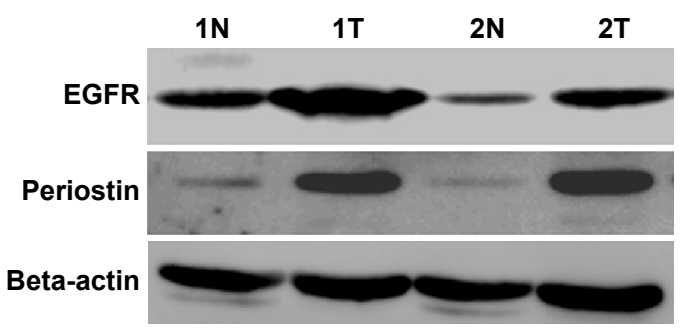

Figure 3 Western blot analysis of PN $(90 \mathrm{kDa})$ and EGFR (I70 kDa) proteins in two fresh-frozen ESCC and corresponding tumor-adjacent normal tissues.

Note: T, ESCC tissues, and N, corresponding tumor-adjacent normal tissues.

Abbreviations: PN, periostin; EGFR, epidermal growth factor receptor; ESCC, esophageal squamous cell carcinoma.

\section{Survival analysis of PN and EGFR}

According to the Kaplan-Meier analysis, disease-free survival (DFS) in patients with high expression of PN was shorter than that in patients with low expression of PN, with DFS time

Table I Relationship between PN, EGFR, and clinicopathological parameters in 83 ESCC patients

\begin{tabular}{|c|c|c|c|c|c|c|c|}
\hline \multirow[t]{2}{*}{ Characteristics } & \multirow[t]{2}{*}{$\mathbf{n}$} & \multicolumn{3}{|l|}{ PN } & \multicolumn{3}{|c|}{ EGFR } \\
\hline & & High & Low & $P$-value & High & Low & $P$-value \\
\hline Gender & & & & 0.130 & & & 0.112 \\
\hline Male & 47 & 30 & 17 & & 34 & 13 & \\
\hline Female & 36 & 17 & 19 & & 20 & 16 & \\
\hline Age (years) & & & & 0.411 & & & 0.649 \\
\hline$<60$ & 26 & 13 & 13 & & 16 & 10 & \\
\hline$\geq 60$ & 57 & 34 & 23 & & 38 & 19 & \\
\hline Tumor length (cm) & & & & 0.044 & & & 0.052 \\
\hline$<5$ & 68 & 35 & 33 & & 41 & 27 & \\
\hline$\geq 5$ & 15 & 12 & 3 & & 13 & 2 & \\
\hline Tumor location & & & & 0.334 & & & 0.285 \\
\hline Upper & 8 & 3 & 5 & & 4 & 4 & \\
\hline Middle & 44 & 25 & 19 & & 28 & 16 & \\
\hline Low & 31 & 19 & 12 & & 22 & 9 & \\
\hline Invasion depth & & & & 0.007 & & & 0.022 \\
\hline pTI & 8 & I & 7 & & 2 & 6 & \\
\hline pT2 & 26 & 13 & 13 & & 16 & 10 & \\
\hline PT3 & 49 & 33 & 16 & & 36 & 13 & \\
\hline Differentiation grade & & & & 0.003 & & & 0.322 \\
\hline Well & 18 & 4 & 14 & & 9 & 9 & \\
\hline Moderate & 51 & 33 & 18 & & 36 & 15 & \\
\hline Poor & 14 & 10 & 4 & & 9 & 5 & \\
\hline Venous invasion & & & & 0.010 & & & 0.066 \\
\hline Yes & 18 & 15 & 3 & & 15 & 3 & \\
\hline No & 65 & 32 & 33 & & 39 & 26 & \\
\hline Lymphatic metastasis & & & & 0.000 & & & 0.000 \\
\hline Yes & 47 & 35 & 12 & & 39 & 8 & \\
\hline No & 36 & 12 & 24 & & 15 & 21 & \\
\hline Stage & & & & 0.000 & & & 0.000 \\
\hline I & 16 & 3 & 13 & & 4 & 12 & \\
\hline II & 31 & 14 & 17 & & 21 & 10 & \\
\hline III & 36 & 30 & 6 & & 30 & 6 & \\
\hline
\end{tabular}

Note: Bold $P$-values are statistically significant.

Abbreviations: PN, periostin; EGFR, epidermal growth factor receptor; ESCC, esophageal squamous cell carcinoma; $\mathrm{PT}$, pathological T stage.
Table 2 The correlation between expression levels of PN and EGFR (cases)

\begin{tabular}{lllll}
\hline PN & \multicolumn{2}{l}{ EGFR expression } & $r$ & P-value \\
\cline { 2 - 3 } & Low $(\mathbf{n}=\mathbf{2 9})$ & High $(\mathbf{n}=\mathbf{5 4})$ & & \\
\hline Low $(n=36)$ & 23 & 13 & 0.531 & 0.000 \\
High $(n=47)$ & 6 & 41 & & \\
\hline
\end{tabular}

Abbreviations: PN, periostin; EGFR, epidermal growth factor receptor; $r$, Spearman's rank correlation coefficient.

of $18.77 \pm 2.45$ months and $44.40 \pm 3.80$ months, respectively ( $P=0.000$; Figure 4A). Moreover, the overall survival (OS) in patients with high expression of $\mathrm{PN}$ was also worse than that in the patients with low expression of PN, with the OS time of 29.19 \pm 2.53 months and 53.69 \pm 2.72 months, respectively $(P=0.000$; Figure 4B). Using EGFR status as a stratification factor, similar results were also found in OS and DFS analyses. DFS and OS in patients with high expression of EGFR were shorter than those in the patients with low expression of EGFR (20.50 \pm 2.12 months vs $46.52 \pm 4.58$ months and $31.80 \pm 2.22$ months vs $54.11 \pm 3.61$ months, respectively; both $P<0.05$; Figure 4C and D).

To deeply understand the role of coexpression of PN and EGFR in prognosis, further analysis was made according to their status of coexpression. The coexpression of $\mathrm{PN}$ and EGFR only contained double-high expression of PN and EGFR in a single cell. Compared with PN or EGFR single marker, the coexpression of PN and EGFR dedicated worse prognosis, which might stratify patients more accurately (both $P<0.05$; Figure 5A and B). Additionally, univariate analysis indicated that prognosis was significantly associated with PN $(P=0.000)$, EGFR $(P=0.000)$, invasion depth $(P=0.000)$, differentiation grade $(P=0.006)$, lymphatic metastasis $(P=0.000)$, and tumor stage $(P=0.000$; Table 3$)$. Moreover, as summarized in Table 4, multivariate Cox analysis indicated that PN expression and EGFR expression were independent prognostic factors. The risk of death for the ESCC patients with low expression of two biomarkers and high expression of single biomarker was 0.243 times $(P=0.000)$ and 0.503 times $(P=0.030)$, respectively, than that for patients with high expression of two biomarkers (Table 5).

\section{Discussion}

Currently, a growing number of studies concentrating on the relationship between the PN and cancers showed that PN was upregulated in various types of cancers. ${ }^{5-10} \mathrm{PN}$ activated signaling pathways functioning in cellular survival, angiogenesis, resistance to hypoxia, tumor invasion, and 
A

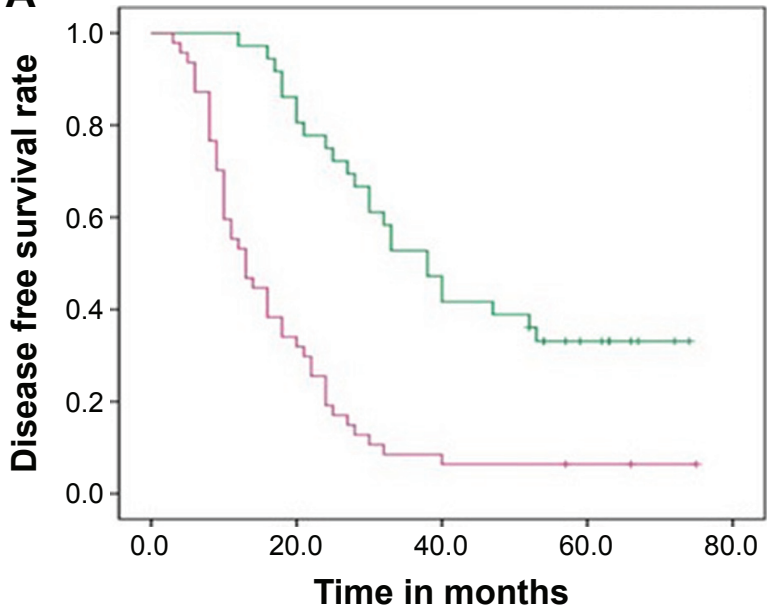

B

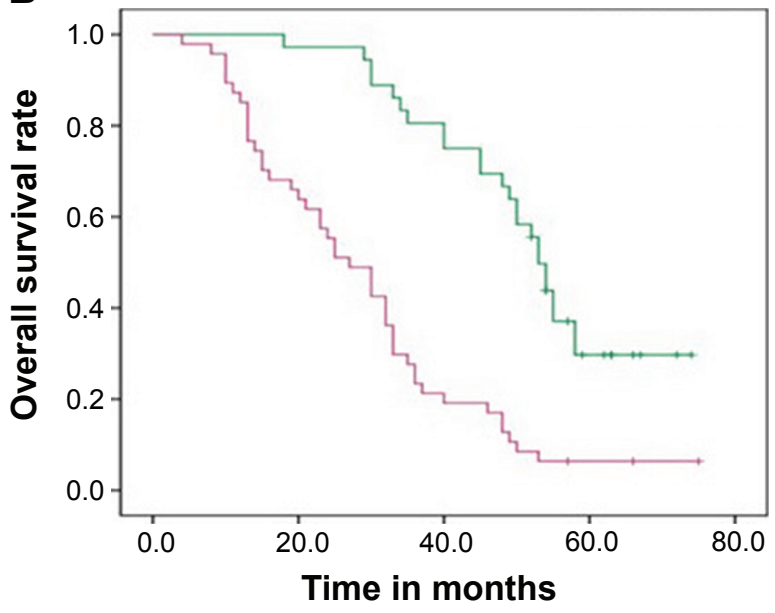

PN expression $\neg$ PN low $\rightarrow$ PN high $\rightarrow$ PN low censored $\rightarrow$ PN high censored

C

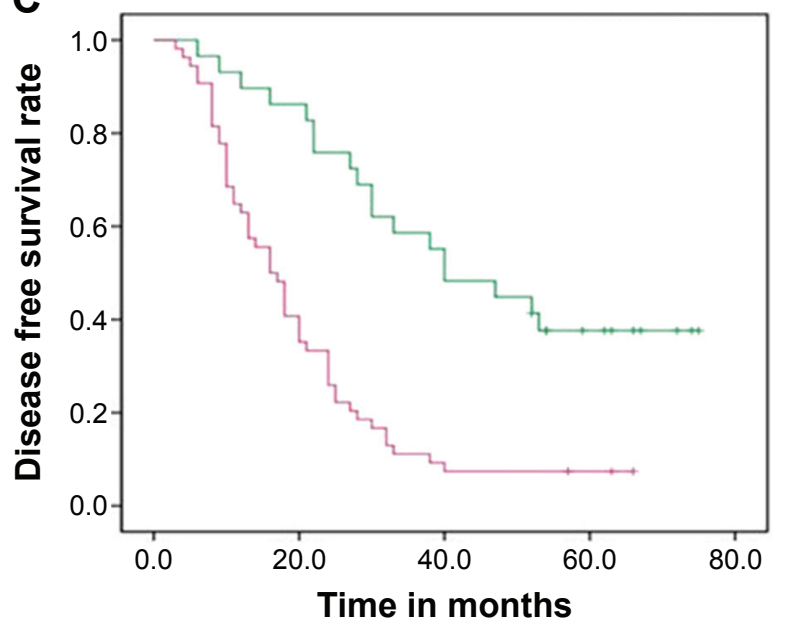

D

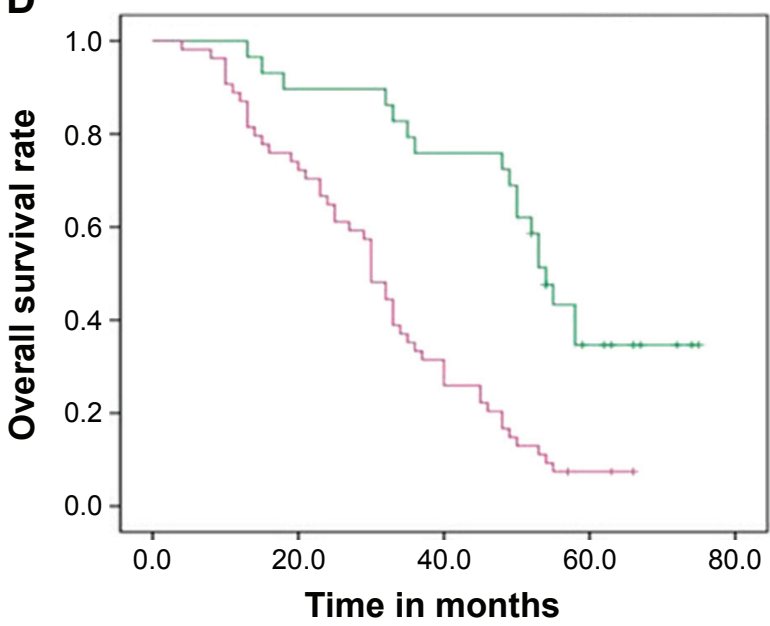

EGFR expression

$\rightarrow$ EGFR low $\rightarrow$ EGFR high $\rightarrow$ EGFR low censored $\rightarrow$ EGFR high censored

Figure 4 Kaplan-Meier survival curves of DFS and OS in the ESCC patients.

Notes: (A) DFS curve of the ESCC patients based on PN expression. (B) OS curve of the ESCC patients based on PN expression. (C) DFS curve of the ESCC patients based on EGFR expression. (D) OS curve of the ESCC patients based on EGFR expression. All $P<0.05$.

Abbreviations: DFS, disease-free survival; OS, overall survival; ESCC, esophageal squamous cell carcinoma; PN, periostin; EGFR, epidermal growth factor receptor.

metastasis. ${ }^{5}$ As for ESCC, accumulative literature provided mounting evidence that $\mathrm{PN}$ played a notable role in tumor invasion, angiogenesis, and remodeling of the extracellular matrix for metastatic colonization. ${ }^{10,18}$ However, there were seldom studies about ESCC focusing on the relationships between PN and EGFR, as well as their coexpression levels in prognostic significance.

In this study, the expression levels of PN were analyzed in the primary ESCC tissues and matched with nontumorous ESCC tissues by IHC. As shown in Figure 1, IHC scores of PN were detectably higher in tumor tissues than those in the tumoradjacent normal tissues. Heidari et al ${ }^{19}$ demonstrated that PN could be evaluated with targeted PET tracer in mice models implanted into TE-11 cells with high PN expression and that the positive rate of strong staining in ESCC was $67.3 \%$. It suggested that the upregulated expression of PN was a common phenomenon in ESCC, which was consistent with previous research, ${ }^{10,18,19}$ and the different expression of PN might help distinguish ESCC from adjacent normal tissues. Luo et $\mathrm{al}^{20}$ found that $\mathrm{PN}$ overexpression in tumor was significantly associated with lymph node metastasis, adventitia invasion, and tumor-node-metastasis (TNM) stage, which had been further confirmed in the research of Wang et al. ${ }^{10}$ However, the relationship between PN 
A

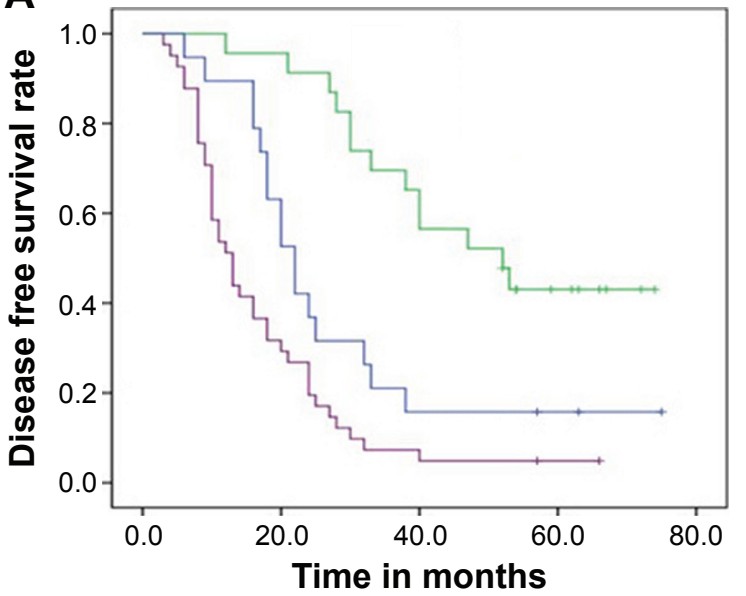

B

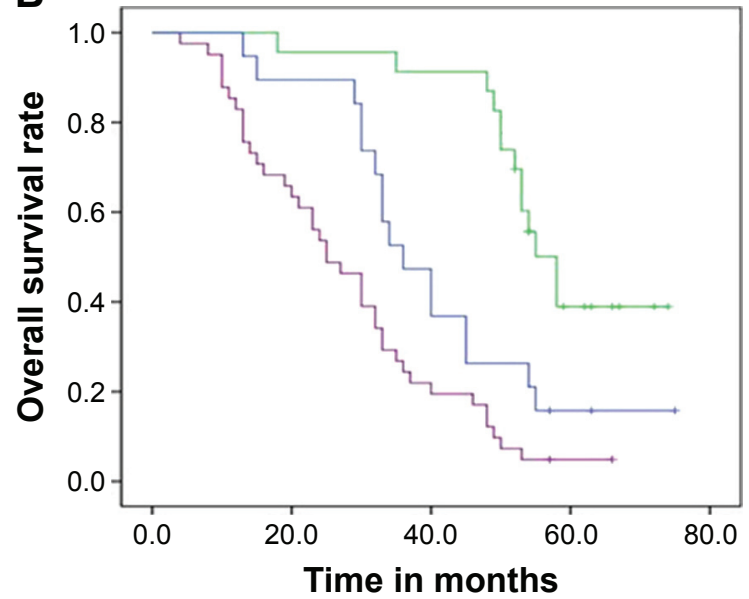

Figure 5 Kaplan-Meier survival curves of DFS and OS based on the coexpression of PN and EGFR.

Notes: (A) DFS curve of ESCC patients based on coexpression of PN and EGFR expression. (B) OS curve of ESCC patients based on coexpression of PN and EGFR expression. All $P<0.05$.

Abbreviations: DFS, disease-free survival; OS, overall survival; PN, periostin; EGFR, epidermal growth factor receptor; ESCC, esophageal squamous cell carcinoma.

expression and tumor differentiation had conflict in the abovementioned reports, which might be caused by the different methods of immunohistochemical evaluation and selection of specimens. In this research, apart from lymphatic metastasis, TNM stage, venous invasion in ESCC, differentiation grade, and tumor length had a significant correlation with the high expression of PN. Further assessment revealed that patients with high expression of $\mathrm{PN}$ had inferior OS and DFS than those with low expression of PN. High expression of PN implies poor prognosis in ESCC, which suggested that PN might support a potential microenvironment for tumor growth and invasion and then affect the prognosis of ESCC patients. ${ }^{5,10}$

The successful treatment of anti-EGFR in non-smallcell lung cancer and colorectal cancer aroused interests of scholars in whether it would be used in esophageal cancer. Although overexpression of EGFR in $40 \%-80 \%$ of esophageal cancer had been reported in previous literature, ${ }^{21,22}$ Jia et a 15 found that the objective response rate of combined nimotuzumab and chemotherapy in ESCC was $55.6 \%$ in the EGFR-high group and $54.1 \%$ in the control group, suggesting that a high expression of EGFR in patients using nimotuzumab could not be an ideal prediction. Preexisting literature showed that an abnormal expression of EGFR was closely related to clinicopathological features such as differentiation, lymphatic metastasis, and stage. ${ }^{21-23}$ Besides lymphatic metastasis and stage, the high expression of EGFR was also significantly correlated with invasion depth in this study; however, there was no relationship between differentiation and EGFR expression. At this stage, the role of EGFR as a predictive factor in ESCC still remained unclear. Some researchers believed that the EGFR overexpression in ESCC patients could predict prognosis. ${ }^{21,23,24}$ In this research, the patients with low expression of EGFR had better OS and DFS than those with high expression of EGFR, which was consistent with the abovementioned studies. However, EGFR as a predictor for ESCC could not be confirmed at EGFR gene or mRNA levels in a previous article. ${ }^{22}$ The inconsistent conclusions might be due to differences in samples and methods for scoring EGFR expression. Larger and further studies are needed to demonstrate the reason for this discrepancy.

To deeply understand the prognostic values of PN and EGFR, the effects of their coexpression were observed on prognosis in the ESCC patients. Interestingly, the risk of death for patients with low expression of two biomarkers and high expression of single biomarker was 0.243 times and 0.503 times, respectively, than that for patients with high expression of two biomarkers. The abovementioned results raised hypothesis that PN combined with EGFR was more effective for prognosis than the single marker in the ESCC patients. By Spearman's rank correlation test, there existed a positive correlation between these two markers with high expression. Compared with the control group, invasion of the 
Table 3 Univariate analysis of characteristics associated with survival time

\begin{tabular}{|c|c|c|c|c|}
\hline \multirow[t]{2}{*}{ Characteristics } & \multicolumn{2}{|l|}{ DFS } & \multicolumn{2}{|l|}{ OS } \\
\hline & $95 \% \mathrm{Cl}$ & $P$-value & $95 \% \mathrm{Cl}$ & $P$-value \\
\hline$\overline{\mathrm{PN}}$ & & 0.000 & & 0.000 \\
\hline Low & $36.956-51.836$ & & $48.360-59.017$ & \\
\hline High & 13.966-23.566 & & $24.236-34.147$ & \\
\hline EGFR & & 0.000 & & 0.000 \\
\hline Low & $37.550-55.485$ & & $47.035-61.181$ & \\
\hline High & $|6.35|-24.649$ & & $27.44 I-36.152$ & \\
\hline Gender & & 0.373 & & 0.331 \\
\hline Male & $21.27 \mid-34.687$ & & $31.852-44.027$ & \\
\hline Female & $24.945-40.166$ & & $35.867-49.036$ & \\
\hline Age (years) & & 0.584 & & 0.546 \\
\hline$<60$ & $19.137-37.152$ & & $29.366-45.160$ & \\
\hline$\geq 60$ & $24.655-36.853$ & & $35.689-46.615$ & \\
\hline Tumor length $(\mathrm{cm})$ & & 0.264 & & 0.220 \\
\hline$<5$ & $25.480-37.151$ & & $35.969-46.099$ & \\
\hline$\geq 5$ & $|5.396-3| .804$ & & $25.374-43.426$ & \\
\hline Tumor location & & 0.649 & & 0.619 \\
\hline Upper/middle & $17.835-30.165$ & & $35.037-46.600$ & \\
\hline Low & $|5.569-26.43|$ & & $30.543-42.877$ & \\
\hline Invasion depth & & 0.000 & & 0.000 \\
\hline $\mathrm{pTI} / \mathrm{pT} 2$ & $36.04|-54.31|$ & & $44.896-59.614$ & \\
\hline $\mathrm{pT} 3$ & $15.947-22.216$ & & $27.001-35.489$ & \\
\hline Differentiation & & 0.005 & & 0.006 \\
\hline grade & & & & \\
\hline Well/moderate & $26.948-38.574$ & & $37.663-47.527$ & \\
\hline Poor & $11.006-22.137$ & & $18.018-35.982$ & \\
\hline Venous invasion & & 0.766 & & 0.673 \\
\hline Yes & I8.797-44.425 & & $29.625-51.486$ & \\
\hline No & $24.072-34.786$ & & $34.77 I-44.482$ & \\
\hline Lymphatic & & 0.000 & & 0.000 \\
\hline metastasis & & & & \\
\hline Yes & | $4.575-22.787$ & & $25.498-34.459$ & \\
\hline No & $35.893-51.616$ & & $45.828-58.365$ & \\
\hline Stage & & 0.000 & & 0.000 \\
\hline I/II & $35.340-49.321$ & & $45.546-56.700$ & \\
\hline III & || $.563-|6.38|$ & & $21.328-29.295$ & \\
\hline
\end{tabular}

Note: Bold $P$-values are statistically significant.

Abbreviations: DFS, disease-free survival; OS, overall survival; $\mathrm{Cl}$, confidence interval; PN, periostin; EGFR, epidermal growth factor receptor; PT, pathological T stage. epithelium into the underlying mesenchymal extracellular matrix showed a minor increase in esophageal cells, which overexpressed EGFR and PN. However, the invasion of ESCC cell lines that overexpressed PN and EGFR was less than those that overexpressed PN and mutant $\mathrm{p} 53 .{ }^{25}$ The underlying detailed mechanisms will be explored in our further studies. Up to now, the available cumulative findings demonstrated that PN binding to the integrins $(\alpha v \beta 3$ and $\alpha v \beta 5)$ regulated phosphatidylinositol-3-kinase-Akt and/or other signaling pathways that promoted tumor invasion and metastasis. ${ }^{26}$ It is worthy of further investigations to verify potential and deep molecular mechanisms of PN and EGFR.

Our study mainly contributed to the prognosis and stratification of ESCC patients by effective molecular markers, in order to offer a potential therapeutic target for ESCC in future. There were some limitations in the present research, which are as follows: it was a retrospective study, the sample size was not large, and the expression levels of PN and EGFR were only examined by IHC and Western blot analyses, without testing mRNA levels and their function by cell experiment in vitro. Hence, future research is still necessary to further elucidate these hypotheses.

\section{Conclusion}

Our research found that both PN and EGFR are overexpressed in ESCC sections compared with paracarcinomatous normal esophageal tissues. Both of them were related to miscellaneous clinicopathologic characteristics and their high expression levels had predictive values on ESCC development and progression. Furthermore, the coexpression of PN and EGFR can be used to predict ESCC patients with different prognoses, which may offer a novel and potential target strategy for ESCC treatment in the future.

Table 4 Multivariate analysis of clinicopathologic features associated with OS and DFS

\begin{tabular}{|c|c|c|c|c|c|c|}
\hline \multirow[t]{2}{*}{ Clinicopathologic features } & \multicolumn{3}{|l|}{ OS } & \multicolumn{3}{|l|}{ DFS } \\
\hline & HR & $95 \% \mathrm{Cl}$ & $P$-value & HR & $95 \% \mathrm{Cl}$ & $P$-value \\
\hline PN (high vs low) & 3.461 & $1.760-6.807$ & 0.000 & 2.392 & $1.210-4.728$ & 0.012 \\
\hline EGFR (high vs low) & 2.246 & $1.060-4.759$ & 0.035 & 2.118 & $1.012-4.435$ & 0.046 \\
\hline Gender (male vs female) & 0.961 & $0.563-1.639$ & 0.883 & 0.814 & $0.477-1.389$ & 0.449 \\
\hline Age ( $<60$ years vs $\geq 60$ years $)$ & 0.599 & $0.339-1.057$ & 0.077 & 0.600 & $0.337-|.07|$ & 0.084 \\
\hline Tumor length $(<5 \mathrm{~cm}$ vs $\geq 5 \mathrm{~cm})$ & 0.877 & $0.443-1.736$ & 0.707 & 1.081 & $0.557-2.100$ & 0.817 \\
\hline Tumor location (upper/middle vs low) & 0.924 & $0.544-1.568$ & 0.769 & 0.868 & $0.507-1.486$ & 0.606 \\
\hline Invasion depth (pT3 vs pTI/pT2) & 3.445 & $1.770-6.705$ & 0.000 & 3.556 & $1.789-7.070$ & 0.000 \\
\hline Differentiation grade (poor vs well/moderate) & 2.211 & $1.132-4.318$ & 0.020 & 1.935 & $1.002-3.736$ & 0.049 \\
\hline Venous invasion (yes vs no) & 0.475 & $0.233-0.968$ & $0.04 I$ & 0.617 & $0.311-1.225$ & 0.168 \\
\hline Lymphatic metastasis (yes vs no) & 0.890 & $0.330-2.399$ & 0.818 & 1.299 & $0.494-3.412$ & 0.596 \\
\hline Stage (III vs I/II) & 3.331 & $1.262-8.780$ & 0.015 & 2.906 & $1.114-7.580$ & 0.029 \\
\hline
\end{tabular}

Note: Bold $P$-values are statistically significant.

Abbreviations: OS, overall survival; DFS, disease-free survival; HR, hazard ratio; $\mathrm{Cl}$, confidence interval; PN, periostin; EGFR, epidermal growth factor receptor; PT, pathological T stage. 
Table 5 Multivariate analysis of correlation between clinicopathologic factors and survival

\begin{tabular}{llllll}
\hline Clinical factors & B & SE & Wald & P-value & HR (95\% CI) \\
\hline Invasion depth & 1.070 & 0.323 & 10.992 & $0.00 I$ & $2.914(1.548-5.484)$ \\
Differentiation grade & 0.883 & 0.319 & 7.667 & 0.006 & $2.418(1.294-4.518)$ \\
Lymphatic metastasis & 0.131 & 0.452 & 0.083 & 0.773 & $1.140(0.470-2.764)$ \\
TNM stage & 1.144 & 0.486 & 5.550 & 0.018 & $3.319(1.212-8.131)$ \\
PN/EGFR & & & 15.117 & 0.001 & \\
PN/EGFR single marker with high expression* & -0.688 & 0.317 & 4.691 & 0.030 & $0.503(0.270-0.937)$ \\
PN/EGFR two markers with low/no expression* & -1.414 & 0.380 & 13.855 & 0.000 & $0.243(0.115-0.512)$ \\
\hline
\end{tabular}

Note: *Compared with PN/EGFR two markers with high expression.

Abbreviations: SE, standard error; HR, hazard ratio; Cl, confidence interval; TNM, tumor-node-metastasis; PN, periostin; EGFR, epidermal growth factor receptor.

\section{Acknowledgment}

This work was partly supported by National Natural Science

Foundation of China (81472329 and 81201906).

\section{Disclosure}

The authors report no conflicts of interest in this work.

\section{References}

1. Pennathur A, Gibson MK, Jobe BA, Luketich JD. Oesophageal carcinoma. Lancet (London, England). 2013;381(9864):400-412.

2. Ohashi S, Miyamoto S, Kikuchi O, Goto T, Amanuma Y, Muto M. Recent advances from basic and clinical studies of esophageal squamous cell carcinoma. Gastroenterology. 2015;149(7):1700-1715.

3. Rustgi AK, El-Serag HB. Esophageal carcinoma. N Engl J Med. 2014; 371(26):2499-2509.

4. Ferlay J, Soerjomataram I, Dikshit R, et al. Cancer incidence and mortality worldwide: sources, methods and major patterns in GLOBOCAN 2012. Int J Cancer. 2015;136(5):E359-E386.

5. Liu AY, Zheng H, Ouyang G. Periostin, a multifunctional matricellular protein in inflammatory and tumor microenvironments. Matrix Biol. 2014;37:150-156.

6. Liu Y, Du L. Role of pancreatic stellate cells and periostin in pancreatic cancer progression. Tumour Biol. 2015;36(5):3171-3177.

7. Zhu M, Fejzo MS, Anderson L, et al. Periostin promotes ovarian cancer angiogenesis and metastasis. Gynecol Oncol. 2010;119(2):337-344.

8. Hong LZ, Wei XW, Chen JF, Shi Y. Overexpression of periostin predicts poor prognosis in non-small cell lung cancer. Oncol Lett. 2013; 6(6):1595-1603

9. Lee YJ, Kim IS, Park SA, et al. Periostin-binding DNA aptamer inhibits breast cancer growth and metastasis. Mol Ther. 2013;21(5): 1004-1013.

10. Wang W, Sun QK, He YF, et al. Overexpression of periostin is significantly correlated to the tumor angiogenesis and poor prognosis in patients with esophageal squamous cell carcinoma. Int J Clin Exp Pathol. 2014;7(2):593-601.

11. Michaylira CZ, Wong GS, Miller CG, et al. Periostin, a cell adhesion molecule, facilitates invasion in the tumor microenvironment and annotates a novel tumor-invasive signature in esophageal cancer. Cancer Res. 2010;70(13):5281-5292.

12. Wong GS, Habibollahi P, Heidari P, et al. Optical imaging of periostin enables early endoscopic detection and characterization of esophageal cancer in mice. Gastroenterology. 2013;144(2):294-297.
13. Li JC, Zhao YH, Wang XY, et al. Clinical significance of the expression of EGFR signaling pathway-related proteins in esophageal squamous cell carcinoma. Tumour Biol. 2014;35(1):651-657.

14. Normanno N, De Luca A, Bianco C, et al. Epidermal growth factor receptor (EGFR) signaling in cancer. Gene. 2006;366(1):2-16.

15. Jia J, Cui Y, Lu M, et al. The relation of EGFR expression by immunohistochemical staining and clinical response of combination treatment of nimotuzumab and chemotherapy in esophageal squamous cell carcinoma. Clin Transl Oncol. Epub 2015 Oct 12.

16. Liang J, E M, Wu G, et al. Nimotuzumab combined with radiotherapy for esophageal cancer: preliminary study of a Phase II clinical trial. Onco Targets Ther. 2013;6:1589-1596.

17. Li Z, Zhang X, Yang Y, et al. Periostin expression and its prognostic value for colorectal cancer. Int J Mol Sci. 2015;16(6):12108-12118.

18. Kashyap MK, Marimuthu A, Peri S, et al. Overexpression of periostin and lumican in esophageal squamous cell carcinoma. Cancers. 2010; 2(1):133-142.

19. Heidari P, Esfahani SA, Turker NS, et al. Imaging of secreted extracellular periostin, an important marker of invasion in the tumor microenvironment in esophageal cancer. J Nucl Med. 2015;56(8):1246-1251.

20. Luo JH, Zhou J, Gao Y. Correlation between periostin and SNCG and esophageal cancer invasion, infiltration and apoptosis. Asian Pac J Trop Med. 2013;6(7):516-519.

21. Wang Q, Zhu H, Xiao Z, et al. Expression of epidermal growth factor receptor is an independent prognostic factor for esophageal squamous cell carcinoma. World J Surg Oncol. 2013;11:278.

22. Zhang L, Wang Y, Bai G, Zhang J, Yang M, Ma X. The relationship between the expression of VEGF, EGFR, and HER-2 mRNA in esophageal squamous cell carcinoma (ESCC) and clinicopathological features of different ethnic groups in Xinjiang. Tumour Biol. 2015;36(12): 9277-9283.

23. Zhang W, Zhu H, Liu X, et al. Epidermal growth factor receptor is a prognosis predictor in patients with esophageal squamous cell carcinoma. Ann Thorac Surg. 2014;98(2):513-519.

24. Jiang D, Li X, Wang H, et al. The prognostic value of EGFR overexpression and amplification in esophageal squamous cell carcinoma. $B M C$ Cancer. 2015;15:377.

25. Wong GS, Lee JS, Park YY, et al. Periostin cooperates with mutant p53 to mediate invasion through the induction of STAT1 signaling in the esophageal tumor microenvironment. Oncogenesis. 2013;2(8):e59.

26. Utispan K, Sonongbua J, Thuwajit $\mathrm{P}$, et al. Periostin activates integrin alpha5beta1 through a PI3K/AKT dependent pathway in invasion of cholangiocarcinoma. Int J Oncol. 2012;41(3):1110-1118. 


\section{Publish your work in this journal}

OncoTargets and Therapy is an international, peer-reviewed, open access journal focusing on the pathological basis of all cancers, potential targets for therapy and treatment protocols employed to improve the management of cancer patients. The journal also focuses on the impact of management programs and new therapeutic agents and protocols on

patient perspectives such as quality of life, adherence and satisfaction. The manuscript management system is completely online and includes a very quick and fair peer-review system, which is all easy to use. Visit http://www.dovepress.com/testimonials.php to read real quotes from published authors.

Submit your manuscript here: http://www.dovepress.com/oncotargets-and-therapy-journal 